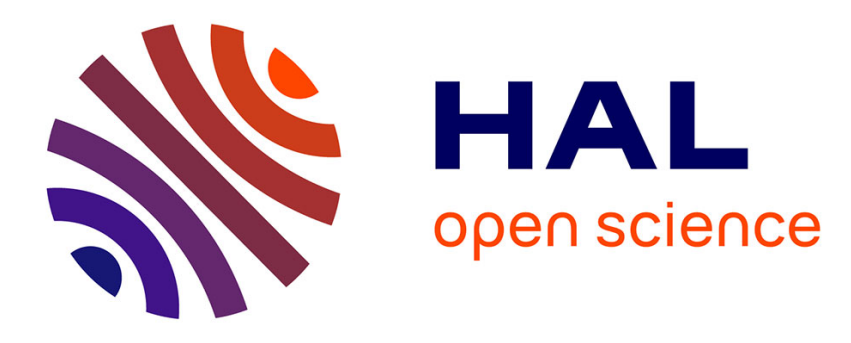

\title{
Microstructures III-V sous pression hydrostatique
} P. Lefebvre, B. Gil, H. Mathieu

\section{To cite this version:}

P. Lefebvre, B. Gil, H. Mathieu. Microstructures III-V sous pression hydrostatique. Revue de Physique Appliquée, 1987, 22 (8), pp.859-866. 10.1051/rphysap:01987002208085900 . jpa-00245624

\section{HAL Id: jpa-00245624 https://hal.science/jpa-00245624}

Submitted on 1 Jan 1987

HAL is a multi-disciplinary open access archive for the deposit and dissemination of scientific research documents, whether they are published or not. The documents may come from teaching and research institutions in France or abroad, or from public or private research centers.
L'archive ouverte pluridisciplinaire HAL, est destinée au dépôt et à la diffusion de documents scientifiques de niveau recherche, publiés ou non, émanant des établissements d'enseignement et de recherche français ou étrangers, des laboratoires publics ou privés. 
Classification

Physics Abstracts

$62.50-73.40 \mathrm{~K}-78.55$

\title{
Microstructures III-V sous pression hydrostatique
}

\author{
P. Lefebvre, B. Gil et H. Mathieu \\ Groupe d'Etudes des Semiconducteurs, Université des Sciences et Techniques du Languedoc, \\ place E. Bataillon, 34060 Montpellier Cedex, France
}

(Reçu le 16 janvier 1987, révisé le 9 avril 1987, accepté le 14 avril 1987)

\begin{abstract}
Résumé. - De récentes mesures de photoluminescence sous pression hydrostatique effectuées sur des puits quantiques III-V de type I montrent un comportement caractéristique de ces microstructures. De manière générale, le coefficient de pression des transitions optiques dans ces structures est inférieur à celui du matériau massif. En comparant les résultats expérimentaux disponibles avec un calcul théorique, nous sommes amenés à distinguer le cas des puits quantiques profonds dans les matériaux à petits gaps de celui des puits peu profonds dans des matériaux à grands gaps. Nous montrons comment, aux effets de la non-parabolicité, sensibles dans ces deux cas, se rajoutent, dans le second cas seulement, ceux de la délocalisation de l'exciton dans les barrières.
\end{abstract}

\begin{abstract}
Recent photoluminescence measurements under hydrostatic pressure, performed on some III-V type I quantum wells, show a characteristic behaviour of these microstructures. Generally speaking, the pressure coefficient of the optical transitions in these structures is lower than that of the bulk material. Comparing the experimental available results with a theoretical calculation, we are led to distinguish between the case of deep quantum wells in narrow gap materials and the one of shallow quantum wells in wide-gap materials. We show how, to the effects of the non parabolicity, present in both cases, the effects of the delocalization of the exciton in the barriers must be added, only in the second case.
\end{abstract}

\section{Introduction.}

L'étude des effets de la pression hydrostatique sur les microstructures (puits quantiques, hétérostructures, super-réseaux, diodes LASER) présente un intérêt incontestable et fait l'objet de nombreuses publications [1-9].

L'avantage de cette technique de perturbation réside dans le fait que des valeurs relativement faibles, de pression permettent d'observer des effets importants. A titre d'exemple, des études de photoluminescence ont montré qu'aux alentours de $40 \mathrm{kbar}$, se produit le croisement des minima $\mathrm{X}$ et $\Gamma$ dans GaAs massif. Plus récemment, d'autres expériences ont montré le même effet dans des structures à puits quantiques $[3,5,8]$, et permis d'accéder aux paramètres encore mal connus que sont les «band-offset », c'est-à-dire en fait, les profondeurs respectives des puits confinant les électrons et les trous. En effet, si dans une structure à puits quantiques, la différence des gaps des matériaux en présence est assez bien connue, la répartition de cette différence entre bande de valence et bande de conduction reste encore un sujet de controverse. Diverses méthodes [7, 10-12] ont été mises en œuvre pour y avoir accès, et il semble bien que l'utilisation de la pression hydrostatique soit un bon moyen d'y parvenir.

D'une manière générale, les études optiques menées à l'aide de la pression hydrostatique sur les puits quantiques ont trait, dans un premier temps aux valeurs des coefficients de pression des transitions observées dans la gamme 0-30 kbar $[2,7,9]$. Dans un second temps, l'intérêt est porté sur le phénomène de croisement cité ci-dessus. Nous nous intéressons ici au domaine des relativement basses pressions dans lequel les travaux les plus récents sur les structures à puits quantiques montrent en particulier que les coefficients de pression des transitions optiques sont toujours inférieurs à ceux du matériau massif.

C'est dans l'explication d'un tel phénomène et de ses corrélations avec les divers paramètres physiques des hétérostructures que réside le but de la présente étude. Celle-ci est articulée comme suit : 
Tout d'abord nous présentons les grandes lignes d'un modèle théorique utilisé pour calculer les énergies des transitions optiques dans les puits quantiques ainsi que leurs coefficients de pression. Dans une seconde partie, nous exposons les résultats expérimentaux et théoriques que nous avons obtenus dans le cas d'un puits profond (1500 meV) dans un matériau à petit gap $(\sim 800 \mathrm{meV}), \mathrm{GaSb}-\mathrm{AlSb}$. Nous établissons ensuite la comparaison entre les résultats de notre modèle avec les expériences de Venkateswaran et al. [3] sur des hétérostructures à puits peu profonds $(\sim 300 \mathrm{meV})$ dans un matériau à grand gap $(\sim 1500 \mathrm{meV}), \mathrm{GaAs}_{-\mathrm{Ga}_{1-x}} \mathrm{Al}_{x} \mathrm{As}$. Enfin, dans la dernière partie, nous tirons les conclusions sur les phénomènes physiques mis en jeu.

\section{Modèle de calcul.}

Notre calcul se place dans le cadre du formalisme de la fonction enveloppe de G. Bastard [13], en assurant la continuité de la fonction enveloppe et de la densité de courant de probabilité aux hétérointerfaces $[9,14]$. Sur la base de ce modèle, nous effectuons un calcul auto-consistant prenant en compte la variation de masse de la particule, due à la nonparabolicité des bandes d'énergie. Nous obtenons ainsi les énergies de confinement des électrons et des trous. Une correction excitonique, donne accès à l'énergie fondamentale de recombinaison électrontrou, c'est-à-dire à l'énergie du photon de luminescence. La valeur de l'énergie de liaison de l'état $1 \mathrm{~s}$ de l'exciton est calculée à partir de sa valeur $R_{0}$ dans le matériau massif dans le cadre du formalisme de G. Bastard [15] établi pour un puits infini, ce qui ne diffère pas de manière dramatique, dans le cas des puits relativement larges étudiés ici, des calculs à puits finis comme ceux de Green et al. [16]. Quand la largeur du puits passe de l'infini à zéro, le Rydberg effectif $R_{0}^{*}$ de l'exciton passe de $R_{0}^{*}$ à $4 R_{0}^{*}$ suivant la courbe présentée sur la figure 2 de la référence [15]. Enfin, les trous lourds et légers étant différemment quantifiés, nous devons considérer successivement les excitons légers et lourds (ceci est illustré sur la figure 3 de la référence [17]). La modélisation de la pression hydrostatique passe par la prise en compte de divers phénomènes : i) diminution de la largeur du puits avec la pression. Son effet est typiquement une augmentation très faible des énergies de confinement. La variation relative de largeur est de l'ordre de $10^{-3} \mathrm{kbar}^{-1}$. Elle est donnée par la théorie de l'élasticité.

ii) variation de la profondeur du puits liée à la différence des coefficients de pression des gaps des matériaux constituant l'hétérostructure ;

iii) augmentation des masses effectives des porteurs due à l'augmentation des gaps. Nous utilisons le modèle de Kane à trois bandes [18] :
Dans les barrières :

$$
\begin{gathered}
\frac{m_{\mathrm{e}}^{\mathrm{b}}(P)}{m_{\mathrm{e}}^{\mathrm{b}}(0)}=\frac{E_{\mathrm{g}}^{\mathrm{b}}+C^{\mathrm{b}} P}{E_{\mathrm{g}}^{\mathrm{b}}} \times \frac{E_{\mathrm{g}}^{\mathrm{b}}+C^{\mathrm{b}} P+\Delta}{E_{\mathrm{g}}^{\mathrm{b}}+\Delta} \times \\
\times \frac{2 \Delta+3 E_{\mathrm{g}}^{\mathrm{b}}}{2 \Delta+3\left(E_{\mathrm{g}}^{\mathrm{b}}+C^{\mathrm{b}} P\right)} \\
\frac{m_{\mathrm{lh}}^{\mathrm{b}}(P)}{m_{\mathrm{lh}}^{\mathrm{b}}(0)}=\frac{E_{\mathrm{g}}^{\mathrm{b}}+C^{\mathrm{b}} P}{E_{\mathrm{g}}^{\mathrm{b}}} \\
\frac{m_{\mathrm{hh}}^{\mathrm{b}}(P)}{m_{\mathrm{hh}}^{\mathrm{b}}(0)}=1
\end{gathered}
$$

Dans le puits :

$$
\begin{gathered}
\frac{m_{\mathrm{e}}^{\mathrm{w}}\left(P, E_{\mathrm{e}}, E_{\mathrm{lh}}\right)}{m_{\mathrm{e}}^{\mathrm{w}}(0,0,0)}=\frac{E_{\mathrm{g}}^{\mathrm{w}}+C^{\mathrm{w}} \cdot P+E_{\mathrm{e}}+E_{\mathrm{lh}}}{E_{\mathrm{g}}^{\mathrm{w}}} \times \\
\times \frac{E_{\mathrm{g}}^{\mathrm{w}}+C^{\mathrm{w}} P+E_{\mathrm{e}}+E_{\mathrm{lh}}+\Delta}{E_{\mathrm{g}}^{\mathrm{w}}+\Delta} \\
\times \frac{2 \Delta+3 E_{\mathrm{g}}^{\mathrm{w}}}{2 \Delta+3\left(E_{\mathrm{g}}^{\mathrm{w}}+C^{\mathrm{w}} P+E_{\mathrm{e}}+E_{\mathrm{lh}}\right)} \\
\frac{m_{\mathrm{lh}}^{\mathrm{w}}\left(P, E_{\mathrm{e}}, E_{\mathrm{lh}}\right)}{m_{\mathrm{lh}}^{\mathrm{w}}(0,0,0)}=\frac{E_{\mathrm{g}}^{\mathrm{w}}+C^{\mathrm{w}} P+E_{\mathrm{e}}+E_{\mathrm{lh}}}{E_{\mathrm{g}}^{\mathrm{w}}} \\
\frac{m_{\mathrm{hh}}^{\mathrm{w}}\left(P, E_{\mathrm{e}}, E_{\mathrm{hh}}\right)}{m_{\mathrm{hh}}^{\mathrm{w}}(0,0,0)}=1
\end{gathered}
$$

où $C^{\mathrm{b}}$ et $C^{\mathrm{w}}$ sont les coefficients de pression des gaps respectifs des barrières et du puits, $P$ est la pression appliquée, $E_{\mathrm{g}}^{\mathrm{b}}$ et $E_{\mathrm{g}}^{\mathrm{w}}$ sont les gaps et $E_{\mathrm{e}}, E_{\mathrm{lh}}$ les énergies de confinement des électrons et des trous légers qui sont, seuls, concernés par le couplage kp. La modification en pression des masses induites par l'augmentation de l'élément de matrice de Kane (de l'ordre de $6 \times 10^{-4} \mathrm{kbar}^{-1}$ en valeur relative) est d'un ordre de grandeur inférieur à celle qui découle de l'ouverture des gaps ; elle peut être raisonnablement négligée.

Les expressions précédentes montrent qu'un calcul auto-consistant est nécessaire pour connaître les valeurs des niveaux d'énergie et des masses. Les équations (1) à (6) montrent que les masses effectives augmentent quand la pression augmente, à la fois dans le puits et dans les barrières. C'est un point intéressant à discuter car cela représente une importante contribution à la variation des coefficients de pression des transitions optiques: dans le puits, l'augmentation de masse provoque un «effondrement " des niveaux vers le fond du puits de potentiel; hors du puits, elle induit une réduction de l'extension spatiale de la fonction enveloppe. Ainsi les variations des masses effectives avec la pression, provoquent une chute des niveaux d'énergie si les masses augmentent plus vite dans les barrières que dans le puits. Dans le cas contraire, les niveaux ont 
tendance à croître. Enfin, considérons la dépendance en pression du Rydberg effectif des excitons. Celleci n'est pas facile à calculer car elle est fonction des variations de la largeur du puits, des masses effectives et de la constante diélectrique.

En considérant, pour la constante diélectrique, une variation donnée par [25] $1 / \varepsilon \mathrm{d} \varepsilon / \mathrm{d} P=\kappa$ le Rydberg tridimensionnel subit. une variation donnée par :

$$
R_{3 \mathrm{D}}^{*}(P)=R_{3 \mathrm{D}}^{*}(0) \exp (-2 \kappa P) \mu(P) / \mu(0)
$$

où $\mu(P)$ et $\mu(0)$ sont les masses effectives réduites des excitons à la pression $P$,et à pression atmosphérique respectivement. Cette variation n'a pas été mesurée pour GaAs, mais a été mesurée par réflectivité à la température de l'hélium liquide dans le phosphure d'indium par Chen et al. [26] ; ces auteurs ont trouvé une variation de $3 \%$ par kilobar, alors que la théorie de la masse effective prévoit $1 \%$. Les expressions utilisées pour les masses des excitons lourds et légers sont explicitées dans [27].

$\mathrm{La}$ variation du rayon de Bohr à trois dimensions est donnée par :

$$
a_{\mathrm{B} 3 \mathrm{D}}^{*}(P)=a_{\mathrm{B} 3 \mathrm{D}}^{*}(0) \exp (\kappa P) \mu(0) / \mu(P) .
$$

En tenant compte de toutes ces variations, nous pouvons calculer les énergies de liaison des excitons lourds et légers pour chaque valeur de la pression.

\section{Puits profonds. Matériaux à petit gap.}

La figure 1 représente l'évolution sous pression hydrostatique du spectre de photoluminescence à $T=2 \mathrm{~K}$ d'un puits quantique GaSb-AlSb d'une épaisseur de $78 \AA$ [7]. Dans la gamme de pression étudiée (0-7 kbar), l'énergie de la transition excitonique associée au puits (QW) augmente linéairement avec la pression, de même que les énergies des transitions e $\mathrm{A}_{0}$ et $\mathrm{A}_{0} \mathrm{X}$ caractéristiques du substrat $\mathrm{GaSb}$ de notre échantillon. Ce dernier a été obtenu par Epitaxie par Jet Moléculaire sur un substrat orienté suivant la direction (001) ; des détails sont donnés dans [19]. Le fait expérimental important ici est que le coefficient de pression de la transition $Q W$ $(14,1 \mathrm{meV} / \mathrm{kbar})$ est inférieur à celui des transitions e $A_{0}$ et $A_{0} X(15,0 \mathrm{meV} / \mathrm{kbar})$.

Nous avons calculé ces coefficients de pression avec les valeurs des divers paramètres données dans le tableau I. Dans un premier temps, nous avons ajusté la répartition bande de valence-bande de conduction de la différence des gaps, afin de retrouver la valeur de la transition mesurée à pression atmosphérique (838 meV). Il s'agit ici d'une méthode indirecte qui nous permet de proposer la valeur suivante : $\Delta E_{\mathrm{v}}=40 \pm 25 \mathrm{meV}$ [7] en assez bon accord avec les valeurs citées dans la littérature $[10,20-22]$ et avec la « règle de l'anion commun »

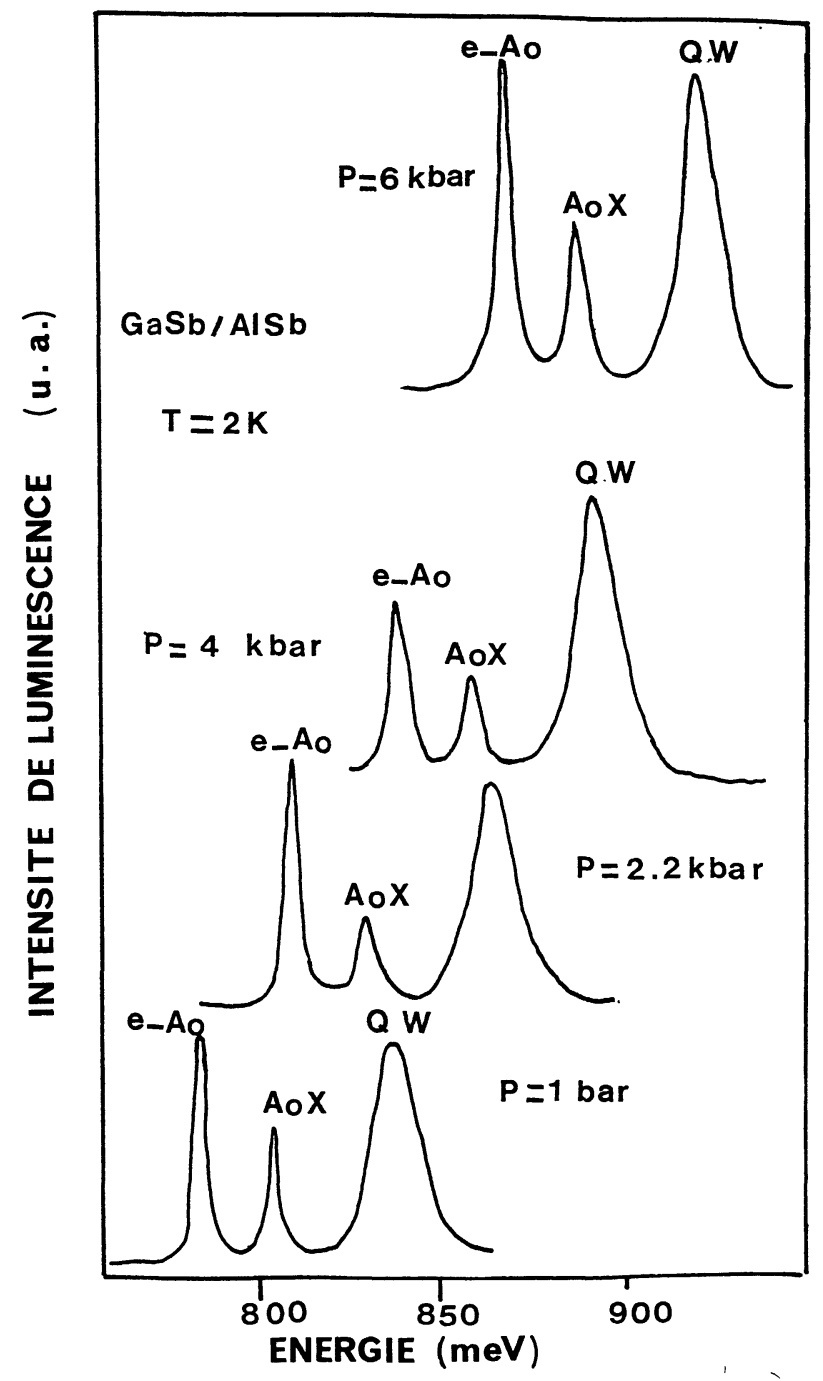

Fig. 1. - Dépendance en pression du spectre de luminescence d'un puits quantique GaSb/AlSb de $78 \AA$ d'épaisseur. $Q W$ représente la recombinaison radiative électrontrou dans le puits quantique. $\mathrm{eA}_{0}$ et $\mathrm{A}_{0} \mathrm{X}$ correspondent au substrat de $\mathrm{GaSb}$.

[Pressure dependence of the luminescence spectrum of a $78 \AA$-wide GaSb/AlSb quantum well. $Q W$ represents the electron-light-hole optically active recombination of the carriers in the quantum well. Both eAo and AoX correspond to the GaSb substrate.]

qui suggère que l'offset de la bande de valence doit rester relativement faible. C'est bien le cas ici, puisque la différence des gaps des deux matériaux en présence est de l'ordre de $1500 \mathrm{meV}$ ce qui entraîne $\delta E_{\mathrm{v}}=0,027 \Delta E_{\mathrm{g}}$. Il est à remarquer que notre échantillon présente la particularité d'être une structure contrainte, en raison du fort désaccord de maille $(0,65 \%)$ qui existe entre le GaSb et l'AlSb. Dans la gamme de pression utilisée, aucune déformation irréversible n'a été produite (en relâchant la pression, on retrouve le spectre de luminescence initialement obtenu à pression atmosphérique). Bien que la couche tampon de AlSb introduite entre le 
Tableau I. - Constantes physiques de GaSb et AlSb. [Physical constants of GaSb and AlSb.]

\begin{tabular}{|l|c|c|}
\hline & GaSb & AlSb \\
\hline$S_{11}\left(10^{-6} \mathrm{bar}^{-1}\right)$ & $1,48\left(^{\mathrm{a}}\right)$ & $1,634\left(^{\mathrm{a}}\right)$ \\
$S_{22}\left(10^{-6} \mathrm{bar}^{-1}\right)$ & $-0,45\left(^{\mathrm{a}}\right)$ & $-0,552\left(^{\mathrm{a}}\right)$ \\
$S_{44}\left(10^{-6} \mathrm{bar}^{-1}\right)$ & $2,23\left(^{\mathrm{a}}\right)$ & $2,38\left(^{\mathrm{a}}\right)$ \\
Paramètre de réseau $\left(10^{-10} \mathrm{~m}\right)$ & $6,0959\left(^{\mathrm{b}}\right)$ & $6,1355\left(^{\mathrm{b}}\right)$ \\
$a(\mathrm{eV})$ & & \\
$b(\mathrm{eV})$ & $-8,30$ & $-5,90$ \\
$\partial E_{\mathrm{G}} / \partial p(\mathrm{meV} /$ Kbar $)$ & -2 & $-1,35$ \\
$m_{0}^{*} \mathrm{e} / m_{0}$ & $15\left(^{\mathrm{c}}\right)$ & $10\left(^{\mathrm{c}}\right)$ \\
$\gamma_{1}$ & $0,041\left(^{\mathrm{b}}\right)$ & $0,110\left(^{\mathrm{b}}\right)$ \\
$\gamma_{2}$ & $11,8\left(^{\mathrm{d}}\right)$ & $4,15\left(^{\mathrm{d}}\right)$ \\
$\gamma_{3}$ & $4,03\left(^{(\mathrm{d}}\right)$ & $1,01\left(^{\mathrm{d}}\right)$ \\
\hline
\end{tabular}

(a) Ref. [28]; (b) Ref. [21]; (c) Ref. [29]; (d) Ref. [30].

substrat de $\mathrm{GaSb}$ et le puits quantique soit relativement mince (1000 ̊̊), sa largeur dépasse l'épaisseur critique au-dessous de laquelle on peut craindre un régime de déformations plastiques [19]. Il paraît donc raisonnable d'estimer que cette couche d'AlSb est entièrement relaxée. Ceci nous conduit à calcu-

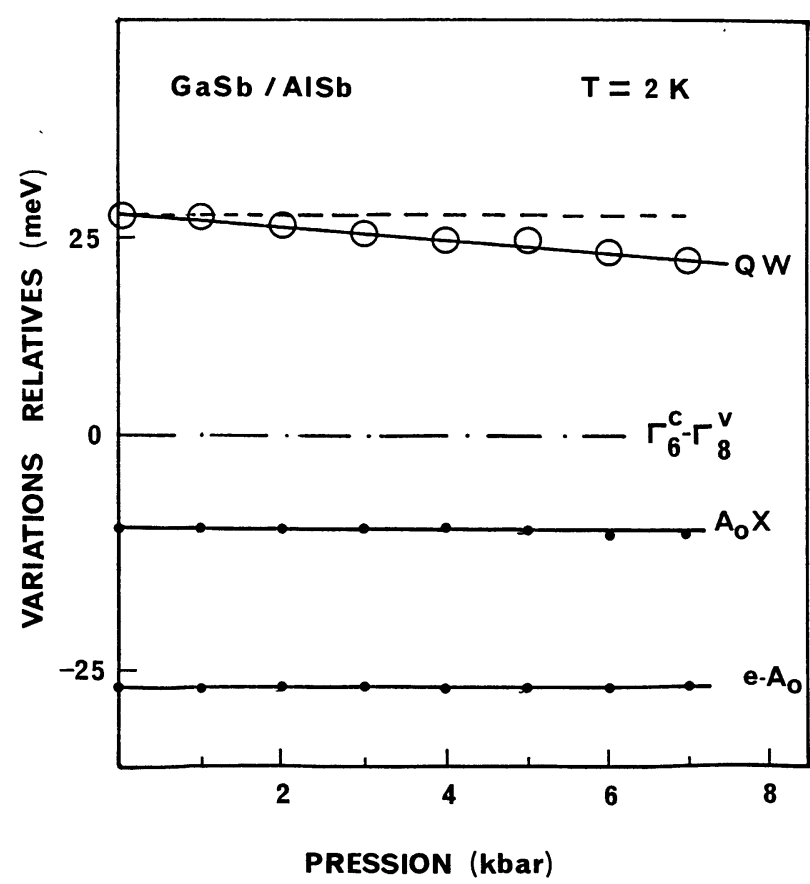

Fig. 2. - Déplacements relatifs des trois pics de luminescence en fonction de la pression. La droite en trait plein $(Q W)$ correspond aux résultats de notre calcul, la droite en pointillés aux prévisions d'un modèle parabolique.

[Relative shift of the three recombination lines versus pressure. The solid line corresponds to the results of our calculation; the dashed one to the predictions of the parabolit . model.] ler, selon la méthode originale proposée par Voi$\sin$ [23], une séparation, en $k=0$, entre les bandes de trous lourds et légers de $45,2 \mathrm{meV}$, due à la contrainte d'interface provoquée par le désaccord des mailles.

$\mathrm{Ce}$ fait a une conséquence très importante. $\mathrm{La}$ transition excitonique fondamentale observée en photoluminescence met en jeu les trous légers de $\mathrm{GaSb}$, et non les trous lourds, comme c'est le cas dans les structures non contraintes du type GaAsGaAlAs [21].

Le résultat de notre calcul est présenté sur la figure 2 , où sont portés les déplacements relatifs des énergies de recombinaison en fonction de la pression. La comparaison sur cette figure entre le calcul ne tenant pas compte de la non-parabolicité des bandes (ligne pointillée) et celui qui la fait intervenir (trait plein) montre que la variation de masse des porteurs, représentée figure 3 , est un paramètre déterminant dans le coefficient de pression de la transition $Q W$. Pour étayer cette hypothèse, nous avons représenté sur la figure 4, les variations calculées d'une part des énergies de confinement des porteurs et d'autre part de l'énergie de liaison de l'exciton. On observe clairement que la variation du

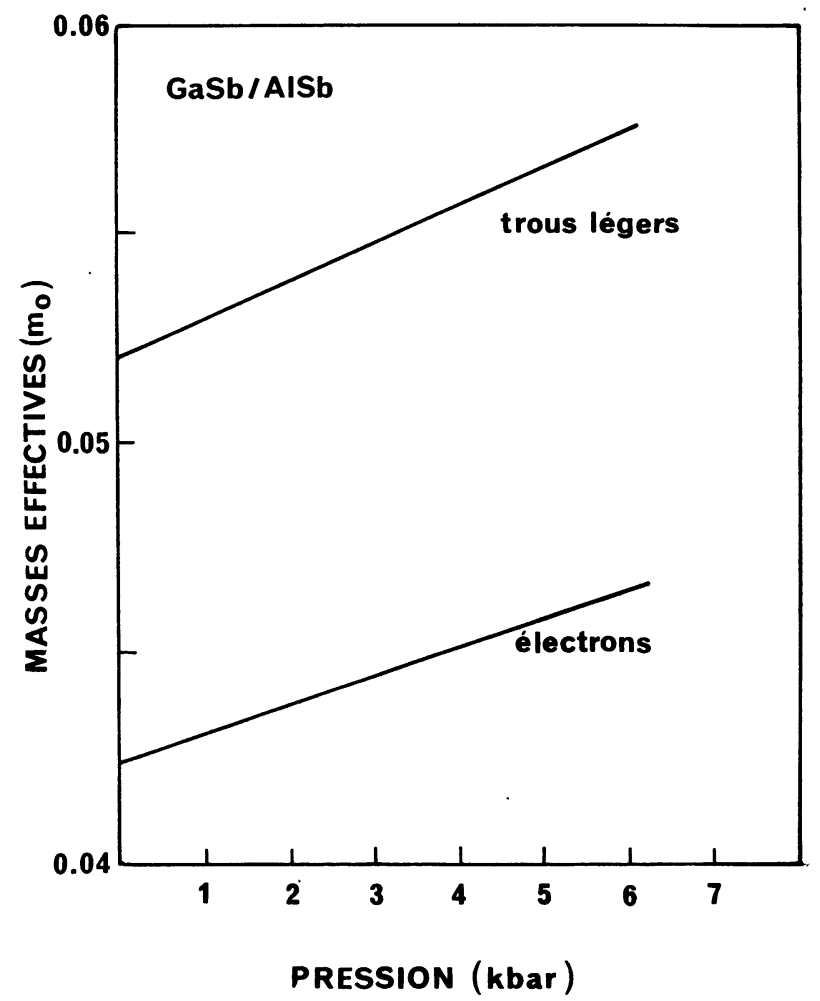

Fig. 3. - Variations calculées, dans le cadre du modèle à trois bandes de Kane, des masses des porteurs dans la couche de $\mathrm{GaSb}$, en fonction de la pression hydrostatique appliquée.

[Computed variations, within the framework of Kane's three-band model, of the carrier-masses in the GaSb layer, versus pressure.] 


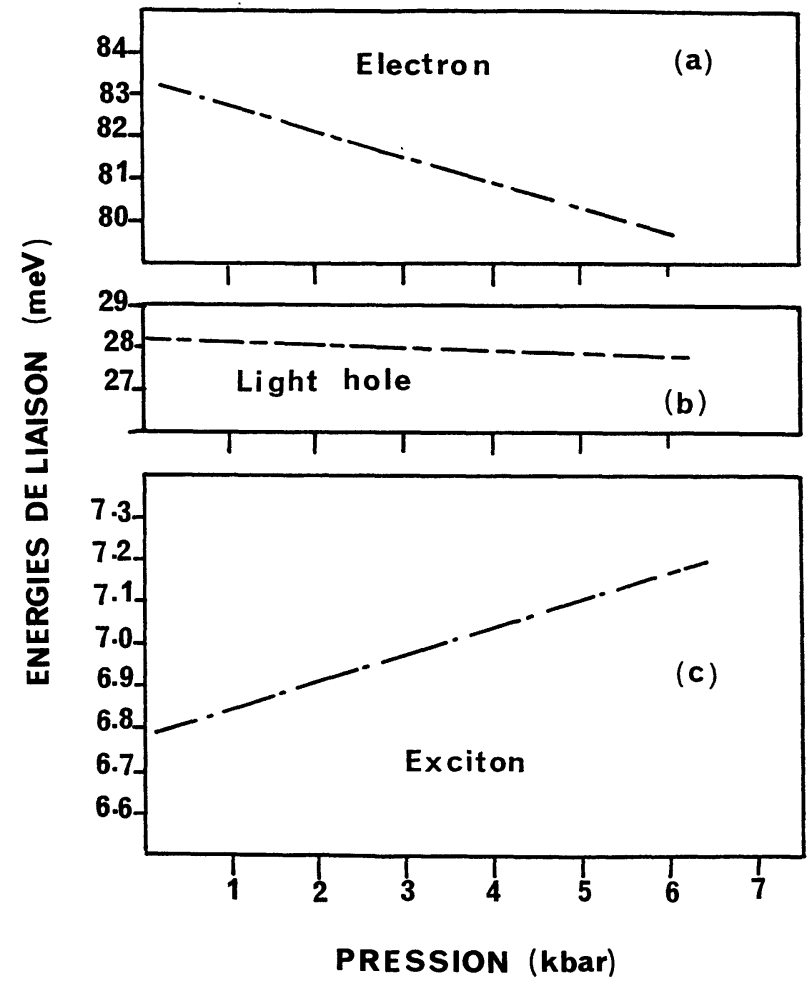

Fig. 4. - Différentes contributions au coefficient de pression de la transition optique dans un puits GaSb-AlSb ( $L_{z}=78 \AA$ ) (a) variation de l'énergie de confinement de l'électron en fonction de la pression, (b) la même figure pour le trou léger, (c) variation du Rydberg effectif dans le puits quantique sous pression.

[Different contributions to the pressure dependence of the optical transition related to the $Q W$. (a) Change in electron confinement versus pressure, (b) the same as (a) but for the light hole, and (c) variation of the effective Rydberg in the $Q W$ under pressure.]

Rydberg effectif dans le puits est inférieure d'un ordre de grandeur à celles des niveaux d'énergie. Autrement dit, l'effet de variation du Rydberg avec la pression est un effet secondaire par rapport à celui de la variation des masses. Remarquons que, dans le cas probable d'une luminescence de caractère extrinsèque de type $\mathrm{p}$ [19], le calcul de Pan et al. [24] montre que la variation de l'énergie de liaison des excitons liés aux accepteurs résiduels de GaSb est, compte tenu de la variation du rapport $\sigma=m_{\mathrm{e}}^{*} / m_{\mathrm{h}}^{*}$ dans la gamme de pression étudiée, très faible. Elle ne peut donc pas contribuer d'une manière mesurable à la valeur du coefficient de pression du puits quantique GaSb-AlSb.

\section{Puits peu profonds. Matériaux à grands gaps.}

Récemment, des études détaillées des effets de la pression hydrostatique sur les niveaux d'énergie des puits quantiques GaAs-GaAlAs ont été publiées [3$6,8]$. Les hétérostructures utilisées sont produites par EJM [3, 4] ou par MOCVD [5, 6, 8]. Nous nous sommes particulièrement intéressés aux travaux de Venkateswaran et al. [3,4] dont les échantillons consistent en une série de plusieurs monopuits de diverses largeurs séparés par de larges barrières de GaAlAs. Des mesures de photoluminescence à basse température et jusqu'à 70 kbar donnent les résultats suivants : i) tous les pics se déplacent vers les hautes énergies quand la pression augmente, ii) les coefficients de pression des transitions excitoniques mettant en jeu les trous lourds et légers sont différents, iii) ces coefficients décroissent lorsque les largeurs des puits diminuent.

Nous avons effectué une série de calculs numériques dans le cas des puits quantiques GaAs-GaA1As, afin d'expliquer les résultats expérimentaux obtenus par Venkateswaran et al. [3, 4] à $80 \mathrm{~K}$. Les valeurs des divers paramètres utilisés dans notre calcul sont résumées dans le tableau II. La figure 5 montre les coefficients de pression des énergies de recombinaison des excitons libres mesurés dans des puits quantiques $\mathrm{GaAs}-\mathrm{Ga}_{0,7} \mathrm{Al}_{0,3} \mathrm{As}$ (cercles blancs et noirs) et les résultats de nos calculs à la fois pour les trous lourds (pointillés) et légers (trait continu).

Tableau II. - Constantes Physiques de GaAs et $\mathrm{Ga}_{1-x} \mathrm{Al}_{x} \mathrm{As}$.

[Physical constants of GaAs and $\mathrm{Ga}_{1-x} \mathrm{Al}_{x} \mathrm{As}$.]

\begin{tabular}{|l|c|c|}
\hline & GaAs & $\mathrm{Ga}_{1-x} \mathrm{Al}_{x} \mathrm{As}$ \\
\hline$S_{11}\left(10^{-6} \mathrm{bar}^{-1}\right)$ & $1,16\left(^{\mathrm{a}}\right)$ & \\
$S_{12}\left(10^{-6} \mathrm{bar}^{-1}\right)$ & $-0,37\left(^{\mathrm{a}}\right)$ & \\
$S_{44}\left(10^{-6} \mathrm{bar}^{-1}\right)$ & $1,67\left(^{\mathrm{a}}\right)$ & \\
$m_{\mathrm{e}}^{*} / m_{0}$ & $0,0665\left(^{\mathrm{b}}\right)$ & $0,0665+0,0174 x\left(^{\mathrm{b}}\right)$ \\
& & $+0,14 x^{2}(x<0,35)$ \\
$\partial E_{\mathrm{G}} / \partial p(\mathrm{meV} / \mathrm{Kbar})$ & $10,7\left(^{\mathrm{c}}\right)$ & $9,8\left(^{\mathrm{d}}\right)$ \\
$\gamma_{1}$ & $7,65\left(^{\mathrm{c}}\right)$ & $4,02\left(^{(}\right)(x=1)$ \\
$\gamma_{2}$ & $2,41\left(^{\mathrm{e}}\right)$ & $0,78\left(^{\mathrm{e}}\right)(x=1)$ \\
$\gamma_{3}$ & $3,28\left(^{\mathrm{e}}\right)$ & $1,55\left(^{\mathrm{e}}\right)(x=1)$ \\
\hline
\end{tabular}

(a) Ref. [31]; (b) Ref. [32]; (c) Ref. [8]; (d) Ref. [3]; (e) Ref. [30].

Deux comportements asymptotiques sont à remarquer. A la limite des grandes largeurs de puits, le caractère bidimensionnel de la microstructure disparaît, ainsi que les effets de quantification, dès que la largeur excède la longueur d'onde de de Broglie des porteurs. Le coefficient de pression de la transition optique tend alors vers celui du GaAs, c'est-à-dire $10,7 \mathrm{meV} / \mathrm{kbar}[3,5,8]$.

A l'opposé, lorsque la largeur du puits devient très faible, le coefficient de pression des structures tend vers le coefficient de pression du gap du matériau constituant la barrière. Dans ce cas, comme le montre la figure 6, les fonctions enveloppes des électrons et des trous sont principalement localisées 


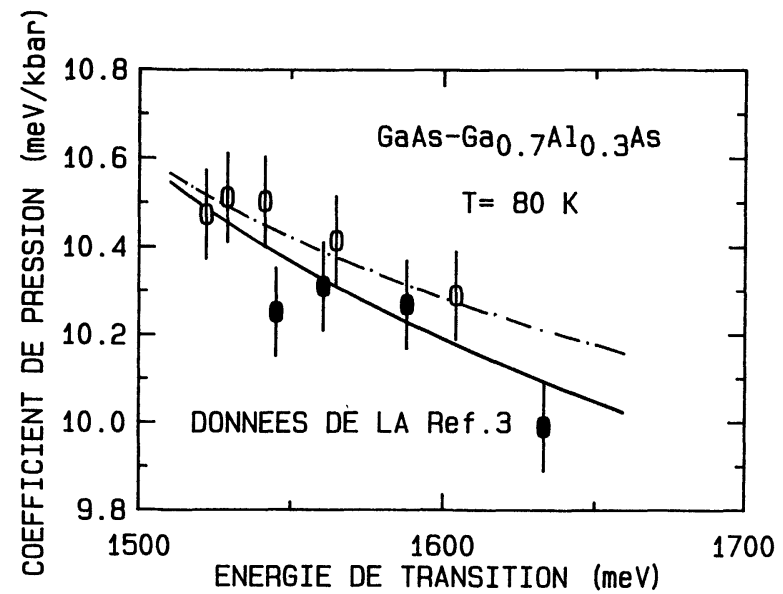

Fig. 5. - Comparaison entre la dépendance en pression d'un puits quantique $\mathrm{GaAs}-\mathrm{Ga}_{0.7} \mathrm{Al}_{0.3} \mathrm{As}$ mesurée à $80 \mathrm{~K}$ (cercles blancs pour les trous lourds et noirs pour les légers) et les résultats de notre calcul (ligne pointillée pour les trous lourds et trait plein pour les trous légers). L'incertitude expérimentale a été représentée par un segment vertical qui correspond aux mesures des coefficients de pression expérimentaux du GaAs et du $\mathrm{Ga}_{0.7} \mathrm{Al}_{0.3} \mathrm{As}$ [3], c'est-à-dire 10,7 $\pm 0,1 \mathrm{meV} / \mathrm{kbar}$ et $9,8 \pm 0,1 \mathrm{meV} / \mathrm{kbar}$.

[Comparison between the pressure dependence of the $\mathrm{Ga}_{0.7} \mathrm{Al}_{0.3} \mathrm{As}$ quantum well measured at $80 \mathrm{~K}$ (open circles for the heavy holes and solid ones for light holes) and the results of that calculation (dash-dotted line for the heavy holes and solid line for the light holes). The experimental uncertainty has been represented by a vertical line, which corresponds to the measurements of the experimental pressure coefficients of $\mathrm{GaAs}$ and $\mathrm{Ga}_{0.7} \mathrm{Al}_{0.3} \mathrm{As}$ [3], that is $10.7 \pm 0.1 \mathrm{meV} / \mathrm{kbar}$ and $9.8 \pm 0.1 \mathrm{meV} / \mathrm{kbar}$ respectively.]

dans les barrières. En effet, pour des largeurs de puits de 10,60 et $110 \AA$, les probabilités de trouver l'électron (le trou léger) dans les barrières sont respectivement de $79 \%, 9,4 \%$ et $2,6 \%(82,6 \%$, $14,8 \%$ et $5,1 \%$ ). Enfin, la figure 7 montre la variation du coefficient de pression de l'exciton léger quantifié en fonction de la probabilité de trouver l'électron dans la couche de GaAs.

On retrouve les comportements asymptotiques des deux cas limites observés précédemment.

\section{Conclusion.}

La tendance générale des transitions optiques dans les puits quantiques de type I est d'avoir un coefficient de pression inférieur à celui du matériau massif. La masse effective des porteurs augmente avec la pression ainsi que leur confinement dans le puits. C'est le cas des puits quantiques contraints GaSb-AlSb pour lesquels les puits de potentiel surtout pour les électrons sont profonds. Dans le cas des puits quantiques GaAs-GaAlAs, où la profondeur des puits n'excède pas quelques centaines de

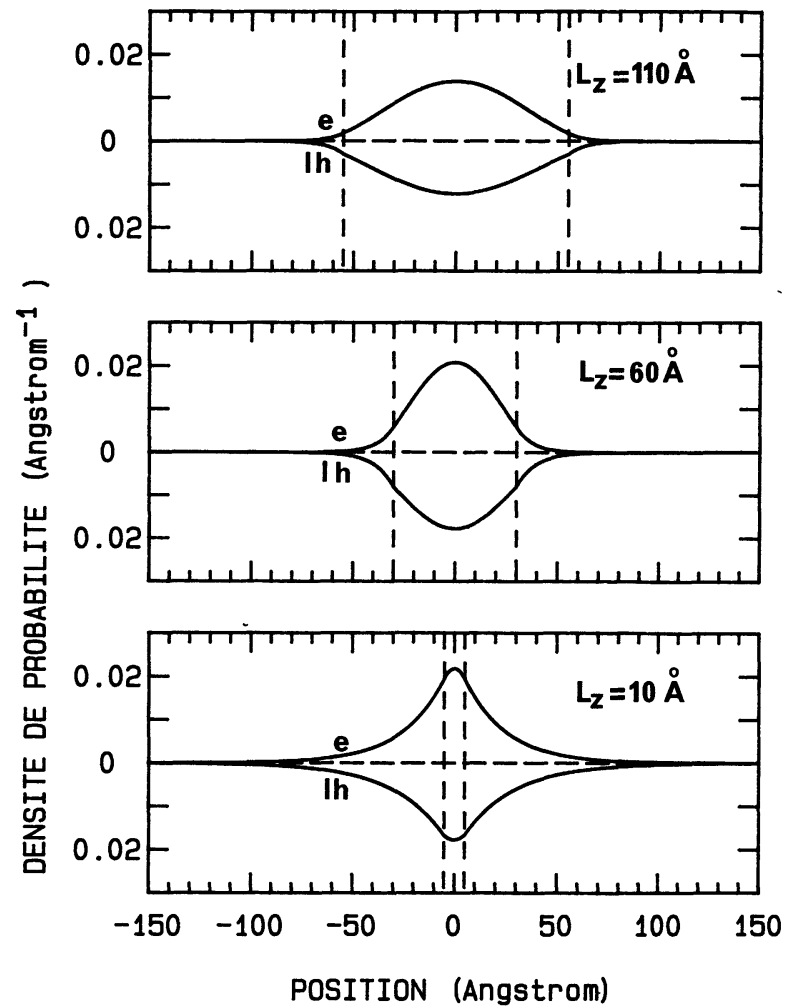

Fig. 6. - Représentation schématique de la densité de probabilité de présence calculée pour l'électron (e) et le trou léger (lh) dans le cas des trois largeurs de puits $\left(\mathrm{GaAs}-\mathrm{Ga}_{0,7} \mathrm{Al}_{0,3} \mathrm{As}\right)$. Les largeurs correspondantes sont (a) : 10, (b) : 60 et (c) : $110 \AA$. Les fonctions d'onde sont plus étendues dans le cas des puits étroits que dans des puits larges. Les probabilités de trouver l'électron dans le puits sont respectivement : $20,99 \%$ (a), 90,63\% (b) et $97,39 \%$ (c). Quant au trou léger on a : 17,34\%(a), $85,20 \%$ (b) et $94,93 \%$ (c).

[Schematic representation of the real-space density of probability obtained for the electron (e) and the light hole (lh) in the case of three widths of the GaAs- $\mathrm{Ga}_{0.7} \mathrm{Al}_{0.3} \mathrm{As}$ QW. The corresponding widths are (a) $10 \AA$, (b) $60 \AA$ and (c) $110 \AA$. The wave function is more delocalized in the space in the case of narrow wells than in the case of wide wells. The probabilities inside the well, $\int\left|F \mathrm{e}^{\mathrm{w}}\right|^{2} \mathrm{~d} z$, are, respectively (a) $20.99 \%$, (b) $90.63 \%$, (c) $97.39 \%$, for the electron. In the case of the light hole, $\int\left|F h^{\mathrm{w}}\right|^{2} \mathrm{~d} z$ gives (a) $17.34 \%$, (b) $85.20 \%$, (c) $94.93 \%$.]

meV, les porteurs sont moins localisés et ont tendance à pénétrer dans les barrières. En pression, la masse effective des porteurs augmente plus vite dans les barrières que dans le puits, ce qui entraîne une diminution du taux de transmission dans les barrières quand l'échantillon est comprimé. Les porteurs sont alors plus confinés et le coefficient de pression de la transition de luminescence prend une valeur intermédiaire entre ceux du GaAs et du GaAlAs.

Quant aux autres effets de la pression, tels que les variations d'épaisseur du puits, du Rydberg effectif, 


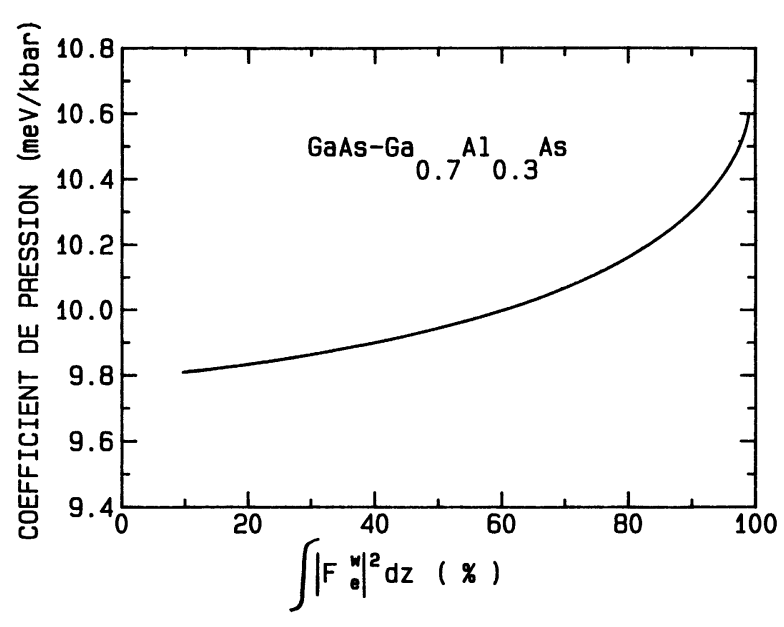

Fig. 7. - Calcul du coefficient de pression de la transition optique électron-trou léger dans un puits quantique $\mathrm{GaAs}-\mathrm{Ga}_{0,7} \mathrm{Al}_{0,3} \mathrm{As}$, en fonction de la probabilité de trouver l'électron dans la couche de confinement (GaAs). Les valeurs asymptotiques de $10,7 \mathrm{meV} / \mathrm{kbar}$ et $9,8 \mathrm{meV} / \mathrm{kbar}$ correspondent au $\mathrm{GaAs}$ et au $\mathrm{Ga}_{0,7} \mathrm{Al}_{0,3}$ As massifs [3].

[Calculation of the pressure coefficient of the quantum well as a function of the probability $\int\left|F \mathrm{e}^{\mathrm{w}}\right|^{2}$ of finding the electron in the GaAs confining layer. The asymptotic values of 10.7 and $9.8 \mathrm{meV} / \mathrm{kbar}$ correspond to bulk GaAs and $\mathrm{Ga}_{0.7} \mathrm{Al}_{0.3} \mathrm{As}$ [3].]

et de l'élément de matrice de Kane, ils semblent se compenser et sont, de toute façon, inférieurs d'un ordre de grandeur aux effets précédemment cités.
En résumé, nous pouvons exhiber la distinction suivante :

i) dans les puits profonds et les matériaux à petit gap, l'exciton est très localisé dans le puits. La faible valeur du coefficient de pression s'explique uniquement par la non-parabolicité des bandes, et donc par l'augmentation des masses en pression;

ii) dans les puits moins profonds et les matériaux à grands gaps, l'augmentation des masses en pression est un peu moins importante et se trouve en concurrence avec l'effet de délocalisation des excitons. Ce fait est particulièrement sensible lorsque l'épaisseur du puits diminue. Le coefficient de pression de la transition tend alors vers celui du gap du matériau constituant la barrière.

\section{Remerciements.}

Nous tenons à remercier M. C. Raisin du Laboratoire d'Etude des Surfaces et Interfaces (USTL) pour la fourniture des échantillons, ainsi que MM. J. Allegre et C. Bousquet sans qui, nous n'aurions pas obtenu nos résultats expérimentaux.

Nos remerciements vont enfin à M. G. Bastard (Groupe de Physique des Solides de l'Ecole Normale Supérieure) et à Mme C. Priester (Laboratoire de physique des solides. Institut Supérieur d'Electronique du Nord), pour leurs conseils et leurs encouragements.

\section{Bibliographie}

[1] Epler, J. E., Kaliski, R. W., Holonyak Jr, N., Peanasky, M. J., Hermannsfeldt, G. A., DRICKAMER, H. G., BURNHAM, R. D. et Thornton, R. L., J. Appl. Phys. 57 (1985) 1495.

[2] Kirchoefer, S. W., Holonyak Jr, N., Hess, K., Meehan, K., Gulino, D. A., Drickamer, H. G., Coleman, J. J. et Dapkus, P. D., J. Appl. Phys. 53 (1982) 6037.

[3] Venkateswaran, U., Chandrasekhar, M., Chandrasekhar, H. R., VoJak, B. A., Chambers, F. A., Meese, J. M., Phys. Rev. B 33 (1986) 8416.

[4] Venkateswaran, U., Chandrasekhar, M., ChandrasekHaR, H. R., Wolfram, T., FisHER, R., MASSElNK, W. T., MORKOC, H., Phys. Rev. B 31 (1985) 4106.

[5] Wolford, D. J., Kuech, T. F., Bradley, J. A., Gell, A. A., NinNo, D. and JARos, M., J. Vac. Sci. Technol. B 4 (1986) 1043.

[6] Weinstein, B. A., HaRK, S. K. et Burnham, R. D., J. Appl. Phys. 58 (1985) 4662.
[7] Lefebvre, P., Gil, B., Allegre, J., Mathieu, H., Chen, Y. et Raisin, C., Phys. Rev. B 35 (1987) 1230.

[8] Wolford, D. J., Proceedings of the XVIIIth International Conference on the Physics of Semiconductors, Stockholm, (1986) and references therein (World Scientific, Singapore) 1987, p. 1115.

[9] Leburton, J. P. et KAHEN, K., Superlattices and microstructures 1 (1985) 49.

[10] Gualtieri, G. J., Schwartz, G. P., Nuzzo, R. G. and Sunder, W. A., Appl. Phys. Lett. 49 (1986) 16.

[11] Meynadier, M. H., Delalande, C., Bastard, G., Voos, M., AleXandre, F. et Lievin, J. L., Phys. Rev., B 31 (1985) 5539.

[12] Menendez, J., Pinczuk, A., Werder, D. J., GosSARD, A. C. et English, J. H., Phys. Rev. B 33 (1986) 8863.

[13] Bastard, G., Phys. Rev. B 24 (1981) 5693.

[14] Altarelli, M., Winter school «Les Houches » (1985). 
[15] Bastard, G., Mendez, E. E., Chang, L. L., [23] Voisin, P., Surf. Sci. 168 (1986) 546.

$$
\text { ESAKI, L., Phys. Rev. B } 26 \text { (1982) } 1974 .
$$

[16] Green, R. L., Bajaj, K. K., Phelps, D. E., Phys. Rev. B 29 (1984) 1807.

[17] Maan, J. C., Belle, G., Fasolino, A., Altarelli, M. et Ploog, K., Phys. Rev. B 30 (1984) 2253.

[18] Kane E. O., Semiconductors and semimetals, Vol. 3 (1966).

[19] Raisin, C., Lassabatere, L., Alibert, C., Girault, B., Abdelfattah, G., Voisin, P., Solid State Commun. 61 (1987) 17.

[20] Voisin, P., Delalande, C., Bastard, G., Voos, M., Chang, L. L., Segmuller, A., Chang, C. A., ESAKI, L., Superlattices and microstructures 1 (1985) 155.

[21] Voisin, P., Delalande, C., Voos, M., Chang, L. L., Segmuller, A., Chang, C. A. et Esaki, L., Phys. Rev. B 30 (1984) 2276.

[22] Mendez, E., Chang, C. A., Takaoka, H., Chang, L. L. et EsAKI, L., J. Vac. Sci. Technol. 1 (1983) 152.
[24] Pan, D. S., Smith, D. L., Mc Gill, T. C., Solid State Commun. 18 (1976) 1557.

[25] Samara, G. A., Phys. Rev. B 27 (1983) 3494.

[26] Chen, Y., Gil, B., Kadri, A., Camassel, J., Allegre, J. et Mathieu, H., Physica 139, 140B (1986) 491.

[27] Priester, C., Allan, G., Lannoo, M., Phys. Rev. B 30 (1984) 7302.

[28] Tuomi, T., Cardona, M., Pollak, F. M., Phys. Status Solidi 40 (1970) 227.

[29] Martinez, G., Optical properties of solids édité par M. Balkanski (North Holland) (1980) 194.

[30] Lawaetz, P., Phys. Rev. B 4 (1971) 3460.

[31] Gavini, A. et Cardona, M., Phys. Rev. B 1 (1969) 672.

[32] Eluani, B., Gibart, P., Portal, J. C. et AulomBARd, R. L., J. Appl. Phys. 58 (1985) 3481. 